\title{
The effects of pre- and post-exercise consumption of multi-ingredient performance supplements on cardiovascular health and body fat in trained men after six weeks of resistance training: a stratified, randomized, double-blind study
}

Michael J Ormsbee ${ }^{1 *}$, Dennison David Thomas', William Kyle Mandler ${ }^{1}$, Emery G Ward ${ }^{1}$, Amber W Kinsey ${ }^{1}$, Lynn B Panton ${ }^{1}$, Timothy P Scheett ${ }^{2}$, Shirin Hooshmand ${ }^{3}$, Emily Simonavice ${ }^{4}$ and Jeong-Su Kim ${ }^{1}$

\begin{abstract}
Background: The cardiovascular (CV) and metabolic health benefits or risks associated with consumption of multi-ingredient performance supplements (MIPS) in conjunction with periodized resistance training (RT) in resistance-trained men are unknown. This population is a major target audience for performance supplements, and therefore, the purpose of this study was to investigate the combined effect of RT and commercially available pre- and post-exercise performance supplements on CV health and body fat in resistance-trained men.

Methods: Twenty-four resistance-trained men completed six weeks (three times/week) of periodized RT while either ingesting SHOT 15-min pre-exercise and SYN immediately post-exercise (multi-ingredient performance supplement group: MIPS) or an isocaloric maltodextrin placebo 15-min pre-exercise and immediately post-exercise (Placebo group). Before and after six weeks of RT and supplementation, resting heart rate (HR), blood pressure (BP), total body fat, android fat, gynoid fat, fat-free mass (FFM) and fasting blood measures of glucose, lipids, nitrate/nitrite (NOx), cortisol and high sensitivity C-reactive protein (hs-CRP) were measured. Statistical analysis was conducted using a one-way ANOVA for baseline differences and a $2 \times 2$ (group $\times$ time) repeated measures ANOVA and Tukey post-hoc tests where appropriate. Significance was set at $p<0.05$.
\end{abstract}

Results: There was no group $\times$ time interaction for HR, BP, blood glucose, lipids, NOx, hs-CRP, cortisol concentrations or body fat. However, there was a time effect where significant decreases in body fat (mean \pm SD; MIPS: $-1.2 \pm 1.2 \%$; Placebo: $-0.9 \pm 1.1 \%$ ), android fat (MIPS: $-1.8 \pm 2.1 \%$; Placebo: $-1.6 \pm 2.0 \%$ ), and gynoid fat (MIPS: $-1.3 \pm 1.6 \%$; Placebo: $-1.0 \pm 1.4 \%)$ for both groups were observed. FFM increased in both groups, and a group $\times$ time interaction was observed with MIPS increasing significantly more than the Placebo group (4.2\% vs. 1.9\%).

Conclusions: Six weeks of MIPS ingestion and periodized RT does not alter CV health parameters or blood indices of health or body fat more than a Placebo treatment in healthy, resistance-trained men. However, MIPS significantly increased FFM more than Placebo.

Keywords: Weight lifting, Supplementation, Regional body fat, Fat-free mass, Health

\footnotetext{
* Correspondence: mormsbee@fsu.edu

'Dept. of Nutrition, Food and Exercise Sciences, The Florida State University,

120 Convocation Way, 430 Sandels Building, Tallahassee, FL 32306, USA

Full list of author information is available at the end of the article
} 


\section{Background}

Nutritional supplements intended for consumption before and after resistance training (RT) to improve performance are extremely popular among young men and athletes $[1,2]$. Given that RT has been shown to increase muscle fiber size and strength in men and women [3] with a concomitant increase in lipolysis and fat oxidation [4], it is not surprising that performance supplements are consumed in conjunction with RT in an attempt to improve body composition and athletic performance. The components of these popular multi-ingredient performance supplements (MIPS; e.g. whey protein, casein protein, branched-chain amino acids (BCAA's), creatine, $\beta$-alanine, caffeine, and L-arginine) have been studied individually for their ergogenic effects [5-8]; however, the health and safety outcomes of these supplements are not well known when consumed in combination in a well-trained population. Caffeine alone has been shown to increase resting blood pressure (BP) acutely [9], and potentially chronically [10]. Systolic blood pressure (SBP) and heart rate (HR) have also been shown to increase during RT with acute caffeine supplementation in resistance-trained men [11]. However, recent studies evaluating supplements that contain caffeine do not report measures of BP or HR [12-14].

Multiple side effects have been demonstrated with the use of MIPS. Shelmadine et al. [12] investigated eighteen untrained men following 28 days of RT (4×/week) when consuming NO-Shotgun ${ }^{\circ}$ (Vital Pharmaceuticals, Davie, FL) containing a blend of protein, BCAA's, creatine, $\beta$-alanine, caffeine, and L-arginine consumed 30 minutes before RT compared to an isocaloric placebo (maltodextrin) and found side effects including dizziness, nausea, headache, and shortness of breath in $44 \%(\mathrm{n}=4$ of 9$)$ of this group's participants. However, 44\% ( $\mathrm{n}=4$ of 9) of participants taking the placebo also experienced side effects of nausea and shortness of breath. Interestingly, the authors did not report $\mathrm{BP}$ or HR data, which may be important given these side effects and media reports portraying these pre- and post-RT products as dangerous. However, fasting blood lipid profile and glucose were not altered in either group. Significant increases were reported in fat-free mass (FFM) with no significant changes in fat mass. This improvement in body composition likely overrides the potential negative consequences to using MIPS supplements for the young athletes targeted by such products $[1,2]$ as anecdotal evidence suggests that young weight training men are most concerned with adding muscle mass.

In a follow-up study, Spillane et al. [13] investigated untrained men and provided NO-Shotgun ${ }^{\circ}$ (Vital Pharmaceuticals, Davie, FL) pre-RT and added consumption NO-Synthesize ${ }^{\circ}$ (Vital Pharmaceuticals, Davie, FL) immediately post-RT and on all non-RT days. The authors reported similar side effects for these untrained participants including nausea, rapid HR, and shortness of breath regardless of being in the MIPS or placebo groups [13]. Again, BP and HR data were not reported but significant $(\mathrm{p}=0.023)$ increases in FFM were found in the supplement group when compared to the placebo group after 28 days (mean \pm SD; Supplement: Day 0, $57.8 \pm 6.4 \mathrm{~kg}$ vs. Day 28, $59.9 \pm 7.6 \mathrm{~kg}$; placebo: Day 0, $56.4 \pm 10.3 \mathrm{~kg}$ vs. Day 28 , $57.0 \pm 9.9 \mathrm{~kg}$ ). In addition, whole blood and serum clinical chemistry markers, regardless of group, remained in the normal ranges in this study [13]. The health implications of MIPS use in trained men, however, deserve further investigation.

Therefore, the purpose of the present study was to evaluate the effects of six weeks of pre- and post-RT MIPS consumption with periodized RT on cardiovascular (CV) and metabolic health and body fat in resistance-trained men. We hypothesized that the inclusion of a pre- and post-RT MIPS with resistance-trained participants would not alter CV markers of health, but would improve cortisol, mood state, and body fat more than a placebo supplement.

\section{Methods \\ Participants}

Twenty-nine healthy, resistance trained ( $\geq 3 \times$ per week; training for $\geq 12$ months) men (mean \pm SD; age, $24.0 \pm 2.3$ years; height, $180.5 \pm 5.8 \mathrm{~cm}$; body mass, $83.7 \pm 0.5 \mathrm{~kg}$; body mass index, BMI, $25.5 \pm 2.2 \mathrm{~kg} \cdot \mathrm{m}^{2}$ ) began this study. However, five (MIPS: $\mathrm{n}=1$; Placebo: $\mathrm{n}=4$ ) withdrew over the course of the six-week protocol (three for personal reasons, one was found to be a smoker, and one did not follow the RT protocol). Participants were non-smokers and had no known existing diseases, musculoskeletal disorders or injuries, uncontrolled hypertension (BP $>140$ / $90 \mathrm{mmHg}$ ), uncontrolled cholesterol/blood lipid levels, use of cholesterol medication, or dairy allergies as assessed by a medical history questionnaire. Those reporting anabolic steroid use on the medical history form were excluded. Use of other performance supplements (e.g. caffeine, $\beta$-alanine, and creatine monohydrate) required a fourweek washout period [15] prior to participation (excluding multivitamins). Participants were instructed not to take any supplement, other than the provided pre- and post-RT supplements, for the duration of the study. All procedures involving human subjects were approved by The Florida State University Human Subjects Institutional Review Board in accordance with the Helsinki Declaration. Written informed consent was obtained prior to participation.

\section{Study design and supplementation protocol}

Participants were randomly assigned to one of two groups in this placebo-controlled, double blind, six-week protocol after being stratified by isometric maximal voluntary contraction strength (dominant quadriceps) to FFM ratio. Participants in the MIPS group consumed $21 \mathrm{~g}$ of NO- 
Shotgun $^{\circledast}$ (SHOT) which contained 72 kcals, 18 g protein, 0 g carbohydrates, $0 \mathrm{~g}$ fat, caffeine $\sim 190 \mathrm{mg}$; containing a proprietary blend of whey protein, BCAA's, creatine, $\beta$-alanine, and L-arginine (Vital Pharmaceuticals, Davie, FL) 15 minutes before RT and $21 \mathrm{~g}$ of NO-Synthesize ${ }^{\bullet}$ (SYN) which contained $\sim 80$ kcals, 20 g protein, 0 g carbohydrate, $0 \mathrm{~g}$ fat; containing a proprietary blend of whey protein, BCAA's, creatine, $\beta$-alanine, and L-arginine (Vital Pharmaceuticals, Davie, FL) immediately after RT. Participants in the Placebo group consumed $21 \mathrm{~g}$ of an isocaloric, flavor-matched maltodextrin placebo 15 minutes before RT and $21 \mathrm{~g}$ immediately post-RT. All supplements were distributed in identical single-serving Ziploc ${ }^{\oplus}$ bags before and after each workout and research personnel watched as each participant consumed the supplement on all training days. For all non-training days, participants were given single-servings of the supplement in Ziploc ${ }^{\circ}$ bags to take home and consume. In the morning for non-training days, MIPS consumed $21 \mathrm{~g} /$ day of SYN and the Placebo group consumed 21 g/day of Placebo. These empty bags were collected and participants were asked to verbally verify supplement compliance on each workout day (our acceptable compliance margin was set to $>80 \%$ to be included in the data analysis).

\section{Laboratory testing}

Laboratory testing took place only before and after the six-week intervention. Profile of mood state [16], HR, BP, body composition, and blood measures took place following a 10-hour fast and a 24-hour restriction of physical activity, caffeine, and alcohol intake at baseline and after six weeks of RT and supplement intervention. Six-week testing (post) took place 48 hours after conclusion of RT to avoid any residual impact of the last training session on dependent variables including blood markers of inflammation (hs-CRP) and catabolism (cortisol) which may be elevated following an acute bout of RT, but not as a result of RT over the entire study period.

\section{Profile of mood states (POMS)}

POMS [16] scores were measured upon arriving to the laboratory and were used to measure mood before and after the six-week intervention. These scores are compiled based on responses to 65 mood descriptors and divided into six categories describing tension, depression, anger, vigor, fatigue, and confusion. In addition, total mood score was calculated. Participants were asked to consider their mood over the previous seven days in their responses.

\section{Heart rate and blood pressure}

Participants rested in a seated position for five minutes after completing the profile of mood states questionnaire. Afterwards, SBP and DPB were measured twice by the same investigator at the same time of day and on the same day and averaged using a manual sphygmomanometer (American Diagnostic Corp., Hauppauge, NY) and stethoscope. HR was measured manually at the radial artery for 60 seconds.

\section{Anthropometrics and body composition}

Height and body mass were measured using a wall-mount stadiometer and electronic scale, respectively (SECA, Birmingham, UK). Total fat mass (FM), FFM, and regional (android and gynoid mass) body composition was determined by dual energy x-ray absorptiometry (DXA; model DPX-IQ; GE Medical Systems; Madison, WI) with participants in the supine position as previously described [17]. Coefficient of variation for body composition analysis using DXA in our laboratory for FFM and FM was 1.9\% and $1.5 \%$ respectively, based on three repeated measures of 10 physically active young men. The quality analysis for the densitometer was conducted on a daily basis using a standard aluminum spine block (phantom) provided by the manufacturer. Measurements of the phantom were within the manufacturer's precision standard with a coefficient of variation $<0.5 \%$.

\section{Cardiovascular and metabolic biomarkers}

Venous blood samples $(10 \mathrm{ml})$ were obtained before and after six weeks of RT and supplementation with MIPS or Placebo. Blood samples were collected in the morning on both laboratory visits as close to the same time as possible into either no preservative (serum) or EDTA-coated (plasma) vacutainer tubes (Becton, Dickinson \& Company, Franklin Lakes, NJ) and centrifuged (IEC CL3R Multispeed Centrifuge, Thermo Electron Corporation, Needham Heights, MA) for 15 minutes at 3500 $\mathrm{rpm}$ at $4^{\circ} \mathrm{C}$. Plasma and serum were then separated and subsequently stored at $-80^{\circ} \mathrm{C}$ in $300 \mu \mathrm{L}$ aliquots until analyzed. Blood was analyzed for total cholesterol (TC), high-density lipoproteins (HDL), TC/HDL ratio, non-HDL (TC-HDL), low-density lipoproteins (LDL), triglycerides (TRG) and blood glucose (Cholestech LDX Analyzer; Cholestech Corp, Hayward, CA). Inter-assay coefficient of variation was $2.1 \%, 4.0 \%, 4.2 \%, 4.1 \%, 4.7 \%$, and $2.3 \%$ for TC, HDL, non-HDL, LDL, TRG, and glucose, respectively.

Total nitrate/nitrite (NOx) and cortisol were determined in duplicate and triplicate, respectively, using commercially available ELISA kits (R\&D Systems, Inc., Minneapolis, MN, USA). High-sensitivity C-reactive protein (hs-CRP) was determined in triplicate using ELISA (IBL International, Inc. Hamburg, Germany). The intra-assay coefficient of variation for NOx, cortisol, and hs-CRP was 3.3\%, 11.3\%, and $7.4 \%$, respectively.

\section{Nutrition analysis}

Dietary analysis was measured using three-day food (two weekdays and one weekend day) records before training 
and during the last week of the study. Participants were asked to maintain their normal eating patterns and habits. FoodWise $^{\mathrm{Tm}}$ dietary analysis software (McGraw-Hill, New York, NY) was used for analysis. Participants were asked to mimic their pre-baseline day of eating prior to posttesting to the best of their ability based off of their dietary food logs.

\section{Reported side effects from supplements}

Participants were asked at each exercise session about any supplement side effects. In addition, they were asked to fill out a questionnaire regarding any side effects at the end of testing (six weeks).

\section{Supplement analysis}

To verify the absence of anabolic steroids, other illicit substances, and concentration of caffeine, the supplements and Placebo were sent to a third party laboratory for analysis (West Chester University of Pennsylvania, West Chester, PA). The samples submitted for analysis were mixed thoroughly and test samples weighed out. Each of these test samples was treated independently and studied in replicate. The analytes of interest were extracted into methanol using liquid-liquid extraction and quantitatively analyzed using gas chromatography-mass spectrometry (GC-MS). A Varian CP 3800 gas chromatograph- Saturn 2000 mass spectrometer with a Rxi-5Sil MS (30 m × 0.25 $\mathrm{mm}$ ID $\times 0.25 \mu \mathrm{m})$ column was used for the analyses. Standards of caffeine purchased from Cerilliant (Round Rock, TX) were used to generate instrument mass-response graphs used for quantitation.

\section{Performance outcomes}

Performance data for this study have been reported previously [18]. Briefly, 1 repetition maximum strength (1RM) of the chest and legs was determined according to National Strength and Conditioning Association (NSCA) 1RM guidelines [19], maximal power was determined with a Wingate test (Monark Ergomedic 874-E, Vansbro, Sweden), and maximal isokinetic and isometric strength was determined with a Biodex System 3 Dynamometer (Shirley, New York).

\section{Resistance training protocol}

All participants completed RT three-days per week for six weeks. The RT protocol was designed to target all of the major muscle groups (chest, back, trapezius, biceps, triceps, shoulders, legs, and abdominals) and was modified from our previous research $[4,20]$ and that of others [13]. This protocol was chosen to allow for periodized progression in weight lifted while decreasing repetitions in order to continually challenge these well-trained athletes. In addition, we opted to follow a three-day protocol rather than a four-day protocol in order to provide the proper supervision of all RT sessions while allowing adequate recovery for the participants. Prior to each exercise session, participants performed a standardized five-minute warm-up on a treadmill. For weeks one and two, three sets of 10 repetitions of each exercise were completed at $70-75 \% 1 R M$. For weeks three and four, three sets of six repetitions of each exercise were completed at $80-85 \%$ 1RM. For weeks five and six, three sets of four repetitions of each exercise were completed at 85-90\% 1RM (Table 1). Day one exercises included flat bench press, incline bench press, chest fly, latissimus pull down, seated row, and shrugs. Day two exercises included leg press, step-ups, leg curl, heel raise, lunge, abdominal crunch, and plank (held 60 seconds). Day three exercises included biceps curl, alternate curl, overhead triceps extension, triceps press down, shoulder press, and reverse fly. Rest intervals were 60-90 seconds between sets and 120 seconds between exercises. If a participant was unable to perform the prescribed weight for an exercise, the weight was adjusted to yield failure at or near the specified number of repetitions. The emphasis placed on consistent lifting form in this study, coupled with researcher supervision from certified personal trainers through the NSCA, helped ensure full participant compliance with training as well as reduce variability due to inter-subject differences or deficiencies in form.

\section{Statistical analysis}

Data were analyzed using a one-way analysis of variance (ANOVA) to determine if there were differences between groups in baseline data. A $2 \times 2$ (group $\times$ time) ANOVA with repeated measures was used to determine the effects of the supplement and Placebo on the dependent measures. A Tukey post hoc analysis was used to identify significant differences when a significant $F$-ratio was obtained. Significance is reported at $\mathrm{p}<0.05$, and all values are reported as means \pm standard deviation (SD). JMP Pro 9 statistical software (SAS Institute Inc., Cary, NC) was used for all analyses.

\section{Table 1 Resistance training protocol}

\begin{tabular}{ccc}
\hline Weeks & RT variables & $\#$ \\
\hline $1-2$ & Sets & 3 \\
& Reps & 10 \\
& Load $(\% 1 R M)$ & $70-75$ \\
$3-4$ & Sets & 3 \\
& Reps & 6 \\
& Load (\%1RM) & $80-85$ \\
$5-6$ & Sets & 3 \\
& Reps & 4 \\
& Load (\% 7 RM $)$ & $85-90$ \\
\hline
\end{tabular}

$\mathrm{RT}$, resistance training. $\mathrm{RM}$, repetition maximum. \#, number. 


\section{Results}

\section{Participant characteristics}

Twenty-four participants completed the study. MIPS $(\mathrm{n}=13)$ had an average age of $23.6 \pm 3.5$ years, height of $180.6 \pm 6.7 \mathrm{~cm}$, and body mass of $83.4 \pm 11.5 \mathrm{~kg}$. Placebo ( $\mathrm{n}=11)$ had an average age of $23.6 \pm 4.6$ years, height of $181.0 \pm 4.7 \mathrm{~cm}$, and body mass of $82.2 \pm 7.2 \mathrm{~kg}$. Groups were not different at baseline (Table 2). Participants were monitored by research personnel with NSCA certifications for all training and supplement consumption on training days. As a result, 100\% compliance was observed for these days with respect to both RT and supplement consumption. On non-training days, participants verbally reported $100 \%$ compliance for supplement ingestion and returned all empty single-serving supplement containers as evidence. As previously reported [18], our periodized RT protocol resulted in significant improvements in 1RM strength in both MIPS and Placebo participants indicating that the protocol was effective in increasing strength.

\section{Profile of mood states}

No group $\times$ time interactions were observed for any profile of mood state variables $(\mathrm{p}>0.05)$. However, there was a main effect of time $(\mathrm{p}=0.014)$ by which training resulted in an increase in vigor regardless of group (MIPS: pre $15.6 \pm 5.8$, post $18.1 \pm 6.9,+16 \%$ vs. Placebo: pre $17.3 \pm 6.9$, post $20.1 \pm 4.7,+16 \%$ ).

\section{Blood pressure and heart rate}

SBP (MIPS: pre $120 \pm 10 \mathrm{mmHg}$, post $117 \pm 14 \mathrm{mmHg}$; Placebo: pre $116 \pm 7 \mathrm{mmHg}$, post $115 \pm 10 \mathrm{mmHg}$ ) and DBP (MIPS: pre $74 \pm 10 \mathrm{mmHg}$, post $72 \pm 10 \mathrm{mmHg}$; Placebo: pre $69 \pm 8$, post $67 \pm 9 \mathrm{mmHg}$ ) were unchanged as a result of training in both groups. Resting HR also did not change as a result of training or supplementation (MIPS: pre $62 \pm 8 \mathrm{bpm}$, post $66 \pm 12 \mathrm{bpm}$; Placebo: pre $59 \pm 6 \mathrm{bpm}$, post $61 \pm 11 \mathrm{bpm})$.

\section{Body composition}

Total body mass (Table 3 ) was increased in both groups $(\mathrm{p}<0.0001)$ but more so in MIPS than Placebo $(\mathrm{p}=0.024)$. FFM (Table 3$)$ was increased in both groups $(\mathrm{p}<0.0001)$;

Table 2 Participant characteristics at baseline $(n=24)$

\begin{tabular}{lccc}
\hline & MIPS & Placebo & $\mathbf{p}$ \\
\hline $\mathrm{n}$ & 13 & 11 & \\
Age (years) & $23.6 \pm 3.5$ & $23.6 \pm 4.6$ & 0.958 \\
Training (years) & $6.1 \pm 3.4$ & $4.5 \pm 3.7$ & 0.291 \\
Height (cm) & $180.6 \pm 6.7$ & $181.0 \pm 4.7$ & 0.856 \\
Body Mass (kg) & $83.4 \pm 11.5$ & $82.2 \pm 7.2$ & 0.767 \\
BMl (kg/m $\left.{ }^{2}\right)$ & $25.4 \pm 2.2$ & $25.1 \pm 2.0$ & 0.664 \\
\hline
\end{tabular}

Data are mean \pm SD. MIPS, multi-ingredient performance supplement. Placebo, carbohydrate placebo. BMI, body mass index. however, the increases were significantly greater with MIPS $(\mathrm{p}=0.025)$. Total body $(\mathrm{p}=0.0005)$, android $(\mathrm{p}=0.0009)$, and gynoid $(\mathrm{p}=0.002)$ fat percentages all significantly decreased over time, with no group by time differences (Figure 1).

\section{Cardiovascular and metabolic biomarkers}

Blood markers of $\mathrm{CV}$ and metabolic health (blood lipid profile, glucose, cortisol, NOx, and hs-CRP) were within normal clinical ranges for the entirety of this study. There were no time or group $\times$ time $(p>0.05)$ effects observed (Table 4) for any of these measures. The sample sizes differed slightly between biomarkers because some participants' samples were not useable or blood was unable to be drawn.

\section{Nutritional analysis}

Data regarding nutritional intake have been reported previously [18]. Briefly, all participants were asked to complete a three-day food record before RT and during the final week of RT, only eight of the participants (MIPS, $\mathrm{n}=5$; Placebo, $\mathrm{n}=3$ ) returned satisfactory food records to the research staff for analysis. However, the participant population included only highly resistancetrained individuals and thus, they verbally reported monotonous eating patterns and were instructed to replicate pre-baseline testing nutritional intake for post-testing. Briefly, total Calories (MIPS, $37.6 \pm 8.3 \mathrm{kcal} / \mathrm{day} / \mathrm{kg}$ vs. Placebo, $25.3 \pm 5.8 \mathrm{kcal} /$ day $/ \mathrm{kg}, \mathrm{p}=0.34$ ), carbohydrate (MIPS, $3.2 \pm 0.7 \mathrm{~g} /$ day $/ \mathrm{kg}$ vs. Placebo, $2.5 \pm 0.8 \mathrm{~g} / \mathrm{day} / \mathrm{kg}$, $\mathrm{p}=0.49$ ), protein (MIPS, $1.9 \pm 0.5 \mathrm{~g} / \mathrm{day} / \mathrm{kg}$ vs. Placebo, $1.4 \pm 0.3 \mathrm{~g} / \mathrm{day} / \mathrm{kg}, \mathrm{p}=0.56$ ), and fat intake (MIPS, $1.8 \pm$ $0.8 \mathrm{~g} / \mathrm{day} / \mathrm{kg}$ vs. Placebo, $1.0 \pm 0.9 \mathrm{~g} /$ day $/ \mathrm{kg}, \mathrm{p}=0.51$ ) for this subgroup $(\mathrm{n}=8)$ was not different at baseline, remained unchanged for the duration of the study and was not different between groups [18].

\section{Performance data}

Performance data have been reported previously [18]. Briefly, no differences existed between groups for training volume and both groups had significant increases in 1RM strength for upper and lower body with no difference between groups (Leg press, MIPS: pre $336 \pm 24 \mathrm{~kg}$, post $418 \pm 25 \mathrm{~kg}, \mathrm{p}<0.001$; Placebo: pre $318 \pm 28 \mathrm{~kg}$, post $429 \pm 29 \mathrm{~kg}, \mathrm{p}<0.001$; Chest press, MIPS: pre $112 \pm 7 \mathrm{~kg}$, post $123 \pm 7 \mathrm{~kg}, \mathrm{p}=0.001$; Placebo, pre $118 \pm 8 \mathrm{~kg}$, post $127 \pm 8 \mathrm{~kg}, \mathrm{p}=0.001)$.

\section{Reported side effects}

Over the six-week study nausea and dry-mouth was reported by one participant in MIPS. Feelings of paresthesia were reported by two participants in MIPS and two participants in the Placebo group. No other side effects were reported. 
Table 3 Body composition at baseline and after six weeks of supplementation and resistance training $(\mathbf{n}=\mathbf{2 4 )}$

\begin{tabular}{|c|c|c|c|c|c|}
\hline Variable & Group & Baseline & Post & Time & Group x Time \\
\hline \multirow[t]{2}{*}{ Body Mass (kg) } & MIPS & $83.4 \pm 11.5$ & $85.8 \pm 11.8$ & $<.0001^{*}$ & $0.024^{\dagger}$ \\
\hline & Placebo & $82.2 \pm 7.2$ & $83.3 \pm 7.7$ & & \\
\hline \multirow[t]{2}{*}{ Fat Free Mass (kg) } & MIPS & $66.8 \pm 9.2$ & $69.6 \pm 9.2$ & $<.0001^{*}$ & $0.025^{\dagger}$ \\
\hline & Placebo & $66.9 \pm 5.3$ & $68.2 \pm 6.0$ & & \\
\hline \multirow[t]{2}{*}{ Body Fat \% } & MIPS & $20.7 \pm 4.0$ & $19.5 \pm 3.8$ & $0.0005^{*}$ & 0.682 \\
\hline & Placebo & $20.2 \pm 5.0$ & $19.2 \pm 4.8$ & & \\
\hline \multirow[t]{2}{*}{ Android Region Fat \% } & MIPS & $22.3 \pm 7.0$ & $20.5 \pm 6.8$ & $0.0009^{*}$ & 0.846 \\
\hline & Placebo & $20.6 \pm 8.7$ & $19.0 \pm 8.7$ & & \\
\hline \multirow[t]{2}{*}{ Gynoid Region Fat \% } & MIPS & $20.8 \pm 4.3$ & $19.5 \pm 3.7$ & $0.0019^{*}$ & 0.666 \\
\hline & Placebo & $21.1 \pm 6.1$ & $20.1 \pm 5.9$ & & \\
\hline
\end{tabular}

Data are mean \pm SD. *Indicates a significant main effect of time. + Indicates a significant difference between groups ( $p<0.05)$. MIPS, multi-ingredient performance supplement $(\mathrm{N}=13)$. Placebo, carbohydrate placebo $(\mathrm{N}=11)$.

\section{Supplement analysis}

Caffeine was only present in the pre-workout supplement (SHOT; $190 \mathrm{mg} \pm 10 \mathrm{mg}$ per $21 \mathrm{~g}$ serving). No additional stimulants were detected in either supplement. No quantifiable amounts of caffeine were detected in the post exercise supplement. These data are in agreement with the supplement fact sheet listed by the manufacturer. No other adulterants such as commonly abused stimulants and known anabolic steroids were detected.

\section{Discussion}

The primary findings from this investigation were that six weeks of MIPS during RT did not alter CV or metabolic health (blood lipid profile, SBP, DBP, HR, glucose, cortisol,
NOx, or hs-CRP) in resistance-trained men. Feelings of vigor and maximal strength increased in both groups regardless of MIPS or Placebo. In addition, six weeks of MIPS in resistance-trained men appear to improve body composition to a greater degree than Placebo. This is in agreement with the results of others who have reported similar findings in untrained participants $[12,13]$. These studies also reported an improvement in muscle mass and body composition in untrained men without any deleterious effect on blood safety markers when consuming MIPS either before or before and after RT for four weeks $[12,13]$. It has not been determined until now, however, whether MIPS impact CV (e.g. blood lipids, BP, and HR) and/or metabolic (e.g. cortisol) health and body composition
A

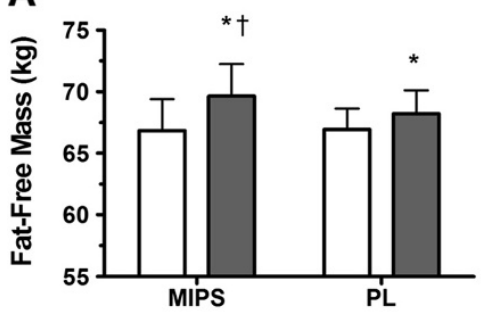

C

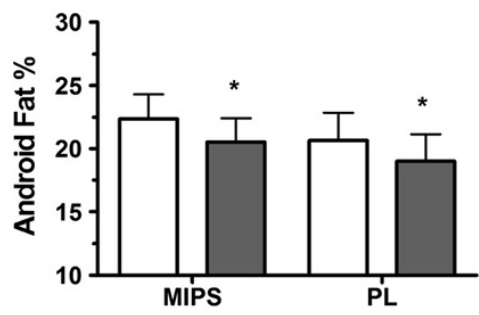

B

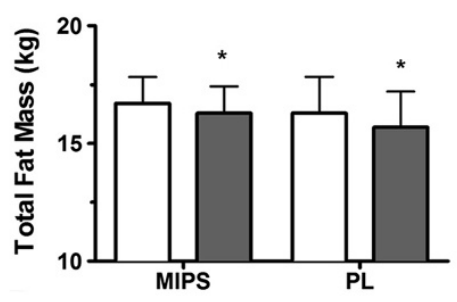

D

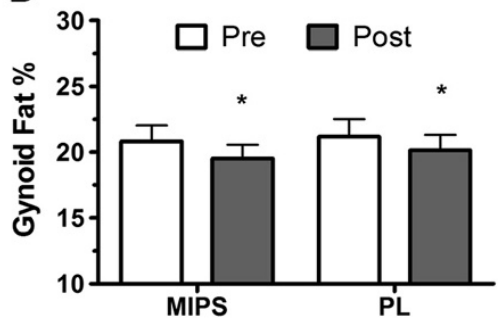

Figure 1 Body composition pre and post six weeks of supplementation and resistance training. A) fat-free mass, B) total fat mass C) android fat percent, and D) gynoid fat percent before and after six weeks of resistance training and supplementation with a multi-ingredient performance supplement (MIPS) or a carbohydrate Placebo pre- and post-exercise in resistance-trained men. White bars, pre-testing; Dark bars, posttesting. ${ }^{*}, \mathrm{P}<0.05$ compared to baseline.,$+ \mathrm{P}<0.05$ compared to Placebo at the same time point. 
Table 4 Serum measurements

\begin{tabular}{|c|c|c|c|c|c|c|}
\hline Variable & Group & $\mathrm{n}$ & Baseline & Post & Time & Group x Time \\
\hline Triglycerides & MIPS & 10 & $90.6 \pm 27.1$ & $118.5 \pm 41.6$ & 0.1124 & 0.571 \\
\hline $\mathrm{mg} / \mathrm{dL}$ & Placebo & 7 & $89.3 \pm 26.9$ & $102.9 \pm 47.8$ & & \\
\hline Total Cholesterol & MIPS & 13 & $150.4 \pm 31.4$ & $145.0 \pm 18.6$ & 0.9464 & 0.273 \\
\hline $\mathrm{mg} / \mathrm{dL}$ & Placebo & 9 & $162.8 \pm 47.5$ & $167.6 \pm 41.5$ & & \\
\hline $\mathrm{HDL}$ & MIPS & 13 & $44.5 \pm 7.4$ & $42.6 \pm 8.2$ & 0.3284 & 0.978 \\
\hline $\mathrm{mg} / \mathrm{dL}$ & Placebo & 11 & $45.0 \pm 13.2$ & $43.1 \pm 6.6$ & & \\
\hline LDL & MIPS & 10 & $89.5 \pm 29.0$ & $82.7 \pm 19.4$ & 0.9253 & 0.201 \\
\hline $\mathrm{mg} / \mathrm{dL}$ & Placebo & 7 & $103.7 \pm 29.7$ & $109.5 \pm 46.6$ & & \\
\hline Non-HDL & MIPS & 13 & $105.9 \pm 30.0$ & $101.2 \pm 21.1$ & 0.9725 & 0.249 \\
\hline $\mathrm{mg} / \mathrm{dL}$ & Placebo & 9 & $117.8 \pm 47.6$ & $122.2 \pm 43.5$ & & \\
\hline Glucose & MIPS & 12 & $94.4 \pm 4.0$ & $97.1 \pm 10.1$ & 0.2655 & 0.821 \\
\hline $\mathrm{mg} / \mathrm{dL}$ & Placebo & 10 & $92.1 \pm 10.4$ & $94.0 \pm 6.8$ & & \\
\hline Cortisol & MIPS & 13 & $123.4 \pm 57.2$ & $123.4 \pm 65.8$ & 0.5071 & 0.503 \\
\hline $\mathrm{nmol} / \mathrm{L}$ & Placebo & 11 & $116.2 \pm 37.1$ & $98.4 \pm 31.5$ & & \\
\hline Endogenous Nitrite & MIPS & 13 & $5.3 \pm 1.6$ & $5.2 \pm 1.1$ & 0.9663 & 0.761 \\
\hline$(\mu \mathrm{mol} / \mathrm{L})$ & Placebo & 11 & $5.3 \pm 1.2$ & $5.4 \pm 1.4$ & & \\
\hline Total Nitrite & MIPS & 13 & $18.4 \pm 18.5$ & $17.9 \pm 18.0$ & 0.7688 & 0.957 \\
\hline$(\mu \mathrm{mol} / \mathrm{L})$ & Placebo & 11 & $20.6 \pm 20.6$ & $20.2 \pm 20.2$ & & \\
\hline Nitrate & MIPS & 13 & $13.1 \pm 6.5$ & $12.7 \pm 11.0$ & 0.7782 & 0.978 \\
\hline$(\mu \mathrm{mol} / \mathrm{L})$ & Placebo & 11 & $15.3 \pm 6.5$ & $14.8 \pm 9.1$ & & \\
\hline \multirow[t]{2}{*}{ Nitrate/Nitrite } & MIPS & 13 & $0.5 \pm 0.4$ & $0.7 \pm 0.7$ & 0.3325 & 0.629 \\
\hline & Placebo & 11 & $0.4 \pm 0.2$ & $0.4 \pm 0.3$ & & \\
\hline hs-CRP & MIPS & 11 & $1.0 \pm 0.87$ & $1.1 \pm 0.97$ & 0.1478 & 0.199 \\
\hline (mg/L) & Placebo & 8 & $0.78 \pm 0.89$ & $2.1 \pm 2.7$ & & \\
\hline
\end{tabular}

Data are mean \pm SD. HDL, high-density lipoprotein; LDL, low-density lipoprotein; Non-HDL, total cholesterol minus HDL; hs-CRP, high-sensitivity C-reactive protein. MIPS, multi-ingredient performance supplement. Placebo, carbohydrate placebo. Sample size between each analyte is slightly different due technical difficulties with certain samples.

in resistance-trained men (a primary marketing target of this class of ergogenic aid). For this reason, we sought to investigate the effects of six weeks of pre- and post-RT MIPS consumption with periodized RT on CV and metabolic health and body fat in resistance-trained men.

Changes in body composition in the current study were dramatic and in some variables, greater than expected based on the current literature. We observed an increase in total body mass in both MIPS $(2.9 \%)$ and Placebo (1.3\%) with MIPS exhibiting a significantly greater gain than Placebo $(\mathrm{p}=0.02)$. Participants in MIPS also increased FFM significantly more than Placebo $(+4.2 \%$ vs. $+1.9 \%$, respectively, $\mathrm{p}=0.025$ ). Similar to Shelmadine et al. [12], these gains mirror increases in FFM measured in untrained men after four weeks of RT with a pre-workout only performance supplement that was the same as was used in the present study (SHOT, $+4.8 \%$ vs. placebo, + 1.7\%). In addition, Spillane et al. [13] reported similar findings of increased FFM in untrained men after four weeks of RT when supplementing both pre- and post-RT with the identical performance supplements used in the present study $(+3.7 \%)$ compared to placebo $(+1.0 \%)$. It is surprising that our findings so closely mimic the results reported in untrained populations despite our participants averaging 4-7 years of RT experience and substantially higher baseline FFM $(+9-13 \mathrm{~kg})$ than participants in the aforementioned studies $[12,13]$. The increase in FFM is most likely due to the creatine and/or $\beta$-alanine in the product; however, due to the proprietary nature of this supplement, the exact amounts of these ingredients are unknown in the product.

Body fat percent decreased significantly in both groups and this was not solely a function of increased FFM. Total body fat decreased by 2.6 and $3.5 \%$ in MIPS and Placebo, respectively. In addition, android (MIPS: -1.8; Placebo: $-1.4 \%$ ) and gynoid fat \% (MIPS: -1.3; Placebo: $-1.0 \%$ ) decreased significantly in both groups. Android fat is highly associated with increased risk of CV mortality and diabetes mellitus and thus, it is notable that our RT 
protocol was successful in reducing both android and gynoid fat. Our findings are supported by research demonstrating that exercise can decrease body fat independent of supplementation [17,21]. Our prior work has shown increased lipolysis with just one bout of RT in resistance-trained men [4] and in overweight/obese sedentary men [20]. A gain in FFM with concurrent decreases in body fat is a highly desirable goal of athletes in many different sports as well as recreational weight-lifters. Our findings support the supplementation of MIPS in combination with periodized RT as an effective method of improving body composition.

Regarding the multitude of individual ingredients in the proprietary blend of SHOT and SYN, including whey protein, BCAA's, creatine, caffeine, $\beta$-alanine, and $\mathrm{L}$-arginine, it is difficult to isolate their specific effects. In fact, some research suggests a synergistic effect of ingredients in the investigated supplements [22-24]. The increases in FFM we observed may likely be explained by studies that used supplementation of creatine with $\beta$-alanine [22] or whey protein and amino acids [23]. Hoffman et al. [22] demonstrated that RT in combination with supplementation of creatine and $\beta$-alanine decreased body fat by $1.2 \%$ through an increase in lean body mass (LBM) $(1.74 \pm 1.72 \mathrm{~kg})$ greater than creatine alone (data not reported) or a carbohydrate placebo $(0.44 \pm 1.62 \mathrm{~kg})$ in collegiate football players. Willoughby et al. [23] observed an increase in FFM of $5.6 \%$ when combining $14 \mathrm{~g}$ of whey and casein protein with $6 \mathrm{~g}$ of free amino acids as compared to a $2.7 \%$ increase with a carbohydrate placebo supplement in untrained men following 10 weeks of heavy RT (3 sets of 6-8 reps with $85-90 \% 1$ RM, $4 \times /$ wk). Perhaps most relevant to the present study, Schmitz et al. [24] recorded $2.4 \%$ and $0.27 \%(\mathrm{p}=0.049)$ increases in $\mathrm{LBM}$ in trained participants ( $\geq$ two years RT experience) supplementing with MIPS with and without BCAA's, respectively. The investigators implemented a nine-week progressive overload RT regimen with participants training four times a week. The two supplements were matched for creatine $(4 \mathrm{~g})$, protein $(7 \mathrm{~g})$, and carbohydrate (39 g). The supplements from the present study include the primary ingredients from these studies [22-24] and alternative ingredients in a proprietary blend that are purported to improve body composition. Our results suggest that the ingredients in SHOT and SYN may be working together to improve body composition greater than the Placebo.

All measured CV and metabolic variables were within normal clinical ranges at baseline and post-six weeks in the present study. Interventions using various intensities of RT with untrained participants have significantly lowered LDL cholesterol in the same six-week span as the present study [25]. While RT may also improve TRG in untrained individuals [26], our participants were experienced with
RT, which likely contributed to the lack of difference measured in these variables. Our findings are supported by previous studies in untrained men $[12,13]$ using SHOT and SYN, which also report no changes in CV health variables after 4 weeks of RT in men of similar age to participants in the current study.

Cortisol is widely accepted as both a catabolic biomarker and an indicator of overall stress. Based on the current literature, we hypothesized a decrease in cortisol concentration in MIPS but measured no changes. Bird et al. [27] reported that both carbohydrate $(6 \%)$ and carbohydrate (6\%) with essential amino acid supplementation (6 g) during an acute bout of RT suppressed the cortisol response in untrained men compared to a placebo. This may have been due to a glucoregulatory effect in which carbohydrate was an adequate source of energy to prevent the need for cortisol to stimulate the liver to release glucose. Sharp and Pearson [28] demonstrated decreased cortisol concentrations in recreationally active young men after three weeks of BCAA supplementation ( $6 \mathrm{~g} /$ day) followed by a fourth week of supplementation and four bouts of RT (three sets of 6-8 repetitions with $80 \% 1 R M$ and 60 seconds between sets and exercises, three lower- and five- upper body exercises). The authors reported significant decreases in cortisol $12 \mathrm{hrs}$ after RT bout two, and 12 and $36 \mathrm{hrs}$ after RT bout four. Because of the low-training status of the participants, the RT was deemed an over-reaching training week. Two factors that may have contributed to the differences observed in our data are the experienced training status of our participants and the slightly later ( $36 \mathrm{vs.} 48 \mathrm{hrs}$ ) post-training cortisol measurements in the present study. Our data indicate training-status may be a determining factor in resting cortisol concentrations with performance nutrition supplementation.

Total nitrate/nitrite (NOx), which is indicative of production of the potent vasodilator, nitric oxide, was also measured to evaluate the impact on CV health. Nitric oxide is synthesized from L-arginine and L-citrulline, two amino acids present in the MIPS in the present study. Nitric oxide has been reported to improve CV health through vasodilation and prevention of lipid build-up on the arterial walls [29]; however, this finding is specific to diseased populations [30]. We recorded no change in resting NOx within or between groups during the intervention, which agrees with most previously published research [13] in healthy individuals. Interestingly, L-arginine has recently been shown to increase blood volume within the muscle (measured with near-infrared spectroscopy) without changing NOx [31]. It is possible that our participants experienced similar changes in blood volume without the detection of any change in NOx. In addition, by design, we sampled blood $48 \mathrm{hrs}$ following the last training bout to measure the impact of supplementation and training over time rather than acute response to RT. 
The inflammatory marker hs-CRP was measured to reflect total body inflammation. Clinical data indicate values of $<1,1-3,>3 \mathrm{mg} / \mathrm{L}$ as low, moderate, and high risk, respectively, for $\mathrm{CV}$ disease. We recorded no significant changes in hs-CRP within or between groups. Kadaglou et al. [32] recently reported no change in CRP following three months of RT $(3 \times /$ week) in type two diabetic patients. Conversely, Sheikholeslami et al. [25] demonstrated significantly lower CRP values in untrained men following six weeks of moderate (45-55\% 1RM) or high intensity (80-90\% 1RM) RT in healthy males compared to a non-intervention control group. The discrepancy in results may be due to the experienced training status of our participants compared to diabetic and untrained individuals. In addition, CRP is notorious for its high intra-subject variability [33] and may have confounded our results.

While RT may independently improve BP in untrained normotensive and pre-hypertensive individuals [26], our data did not indicate changes in BP, perhaps due to our participants' RT history. Similarly, we saw no changes in $\mathrm{HR}$, likely due to their training status. Research indicates that caffeine, a central ingredient in SHOT, the pre-exercise MIPS used in the present study, can increase HR, SBP, and DBP acutely [9]. Energy drinks with less caffeine than SHOT (80 vs $\sim 190 \mathrm{mg}$, respectively) have been reported to elevate BP up to $24 \mathrm{hrs}$ after consumption in nine healthy (aged 18-45 years), nonsmoking, normotensive men $(n=4)$ and women $(n=5)$ who self-reported to consume between four and $379 \mathrm{mg} /$ day of caffeine (four participants reported habitual caffeine intake) [34]. However, some research suggests chronic coffee drinkers are at a decreased risk for developing CV disease [35]. No published research indicates the $\mathrm{HR}$ and BP response to caffeine-containing MIPS.

Regarding mood states, RT elicited an increase in vigor for both MIPS and Placebo. This indicates that it was unlikely our participants were overtrained at the post-testing time point. Bresciani et al. [36] reported a non-significant decrease in vigor and a significant decrease in total mood as being indicative of overtraining in active men.

Resistance-trained men appear to tolerate SHOT and SYN better than their untrained counterparts. Indeed, Shelmadine et al. [12] reported feelings of dizziness, nausea, headache, rapid HR, shortness of breath, and nervousness after consuming SHOT in untrained men. Similarly, Spillane et al. [13] reported select untrained participants experiencing the same side-effects while supplementing with both SHOT and SYN. Only one individual taking SHOT and SYN in the present study reported feeling nauseous while two others reported feelings of paresthesia (although two people in Placebo also reported feeling paresthesia). It is probable that individuals experienced in RT are also experienced with a variety of MIPS and may be more tolerant or less likely to report side effects if any were present.

\section{Limitations}

The present study was limited by the accuracy of self-reported dietary intake and supplement consumption on non-training days. However, our research staff collected empty supplement single-serving Ziploc ${ }^{\bullet}$ bags three times per week in an effort to verify compliance and all participants verbally reported no change in eating patterns. Due to the small sample of useable nutrition logs, it is possible that we did not have a representative sample to determine no differences between groups. However, all participants were questioned personally and reported no change in dietary habits for the duration of the six-week study. In addition, differences in prior training status of our participants may have limited our findings; however, even though MIPS had a longer training history (Table 2), they still were able to increase FFM more than Placebo. In addition, those in the MIPS group may have been able to recognize the feelings from the pre-workout supplement that contained caffeine and $\beta$-alanine and known they were in the supplement group. Moreover, participants were not monitored 24 hours per day and thus may have been able to discuss how the supplement felt to them. However, all training was completed with limited contact to other participants and any interaction would have been outside of workout and/or laboratory visits. Lastly, we do not have reliability data for the POMS assessment used in this study.

\section{Conclusions}

Considering the popularity of MIPS, it is important to confirm their safety and efficacy. Six weeks of MIPS before and after RT does not alter HR, SBP, DBP, fasted blood lipids, glucose, cortisol, NOx, or hs-CRP in healthy resistance-trained men. However, FFM was increased to a greater extent in MIPS than Placebo and improvements occurred in regional body fat and feelings of vigor, regardless of treatment group. Future studies investigating the effects of MIPS on cardiometabolic health variables and body composition should consider closely controlling diet, extending supplementation beyond six weeks, and including female participants and individuals older than 40 years of age.

\section{Competing interests}

This study was supported by an independent research grant and product donation from VPX Pharmaceuticals (Davie, FL). None of the authors had financial or other interests concerning the outcomes of the investigation. The authors declare that they have no competing interests. 


\section{Authors' contributions}

$\mathrm{MJO}$ conceived and designed the study, secured funding for the project, provided oversight of data collection, analysis, biochemical assays, and manuscript preparation. DDT carried out all subject recruitment, data collection, exercise training, immunoassays, and assisted with manuscript preparation. WKM carried out data collection, participant recruitment, and exercise training. AWK, EGW, SH, and ES helped with data collection and analysis. LBP, TPS, and JSK provided assay support, and insight into drafting the study design and manuscript. All authors read and approved the final manuscript.

\section{Acknowledgements}

The authors would like to thank the participants and our personal training staff for their dedication and participation in this study. We would like to thank Jim and Joe Burtoft for generously allowing us to use their Gold's Gym facilities in Tallahassee, FL. We would also like to thank C. Riley, M. Joshi, S. Little, and J. Washburn for analytical support.

\section{Author details}

'Dept. of Nutrition, Food and Exercise Sciences, The Florida State University, 120 Convocation Way, 430 Sandels Building, Tallahassee, FL 32306, USA.

${ }^{2}$ Dept. of Health and Human Performance, College of Charleston, 66 George St, Charleston, SC 29424, USA. ${ }^{3}$ School of Exercise \& Nutritional Sciences, San Diego State University, San Diego, CA 92182, USA. ${ }^{4}$ Dept. of Kinesiology, Georgia College and State University, Milledgeville, GA 31061, USA.

Received: 6 February 2013 Accepted: 25 April 2013

Published: 16 May 2013

\section{References}

1. Young CR, Stephens MB: Sports and nutritional supplement use in USMC recruits: a pilot study. Mil Med 2009, 174:158-161.

2. Maughan RJ, Depiesse F, Geyer H: International Association of Athletics Federations: The use of dietary supplements by athletes. J Sports Sci 2007, 25(Suppl 1):S103-13.

3. Kosek DJ, Kim JS, Petrella JK, Cross JM, Bamman MM: Efficacy of 3 days/wk resistance training on myofiber hypertrophy and myogenic mechanisms in young vs. older adults. J Appl Physiol 2006, 101:531-544.

4. Ormsbee MJ, Thyfault JP, Johnson EA, Kraus RM, Choi MD, Hickner RC: Fat metabolism and acute resistance exercise in trained men. J Appl Physiol 2007, 102:1767-1772.

5. Cribb PJ, Williams AD, Carey MF, Hayes A: The effect of whey isolate and resistance training on strength, body composition, and plasma glutamine. Int I Sport Nutr Exerc Metab 2006, 16:494-509.

6. Cramer JT, Stout JR, Culbertson JY, Egan AD: Effects of creatine supplementation and three days of resistance training on muscle strength, power output, and neuromuscular function. J Strength Cond Res 2007, 21:668-677.

7. Green JM, Wickwire PJ, McLester JR, Gendle S, Hudson G, Pritchett RC, Laurent CM: Effects of caffeine on repetitions to failure and ratings of perceived exertion during resistance training. Int I Sports Physiol Perform 2007, 2:250-259.

8. Kendrick IP, Harris RC, Kim HJ, Kim CK, Dang VH, Lam TQ, Bui TT, Smith M, Wise $J A$ : The effects of 10 weeks of resistance training combined with beta-alanine supplementation on whole body strength, force production, muscular endurance and body composition. Amino Acids 2008, 34:547-554.

9. Karatzis E, Papaioannou TG, Aznaouridis K, Karatzi K, Stamatelopoulos K, Zampelas A, Papamichael C, Lekakis J, Mavrikakis M: Acute effects of caffeine on blood pressure and wave reflections in healthy subjects: should we consider monitoring central blood pressure? Int J Cardiol 2005, 98:425-430.

10. Vlachopoulos C, Panagiotakos D, loakeimidis N, Dima I, Stefanadis C: Chronic coffee consumption has a detrimental effect on aortic stiffness and wave reflections. Am J Clin Nutr 2005, 81:1307-1312.

11. Astorino TA, Rohmann RL, Firth K, Kelly S: Caffeine-induced changes in cardiovascular function during resistance training. Int I Sport Nutr Exerc Metab 2007, 17:468-477.

12. Shelmadine B, Cooke M, Buford T, Hudson G, Redd L, Leutholtz B, Willoughby DS: Effects of 28 days of resistance exercise and consuming a commercially available pre-workout supplement, NO-Shotgun(R), on body composition, muscle strength and mass, markers of satellite cell activation, and clinical safety markers in males. J Int Soc Sports Nutr 2009, 6:16.
13. Spillane M, Schwarz N, Leddy S, Correa T, Minter M, Longoria V, Willoughby DS: Effects of 28 days of resistance exercise while consuming commercially available pre- and post-workout supplements, NO-Shotgun and NO-Synthesize on body composition, muscle strength and mass, markers of protein synthesis, and clinical safety markers in males. Nutr Metab (Lond) 2011, 8:78.

14. Smith AE, Fukuda DH, Kendall KL, Stout JR: The effects of a pre-workout supplement containing caffeine, creatine, and amino acids during three weeks of high-intensity exercise on aerobic and anaerobic performance. J Int Soc Sports Nutr 2010, 7:10.

15. McKenna MJ, Morton J, Selig SE, Snow RJ: Creatine supplementation increases muscle total creatine but not maximal intermittent exercise performance. J Appl Physiol 1999, 87:2244-2252.

16. McNair DM, Lorr M, Droppleman LF: Manual for the profile of mood states. San Diego, CA: Educational and Industrial Testing Services; 1971.

17. Arciero PJ, Gentile CL, Martin-Pressman R, Ormsbee MJ, Everett M, Zwicky L, Steele CA: Increased dietary protein and combined high intensity aerobic and resistance exercise improves body fat distribution and cardiovascular risk factors. Int J Sport Nutr Exerc Metab 2006, 16:373-392.

18. Arciero PJ, Gentile CL, Pressman R, Everett M, Ormsbee MJ, Martin J, Santamore J, Gorman L, Fehling PC, Vukovich MD, Nindl BC: Moderate protein intake improves total and regional body composition and insulin sensitivity in overweight adults. Metab Clin Exp 2008, 57:757-765.

19. Hoffman J, Ratamess N, Kang J, Mangine G, Faigenbaum A, Stout J: Effect of creatine and beta-alanine supplementation on performance and endocrine responses in strength/power athletes. Int I Sport Nutr Exerc Metab 2006, 16:430-446.

20. Ormsbee MJ, Choi MD, Medlin JK, Geyer GH, Trantham LH, Dubis GS, Hickner RC: Regulation of fat metabolism during resistance exercise in sedentary lean and obese men. J Appl Physiol 2009, 106:1529-1537.

21. Ormsbee MJ, Mandler WK, Thomas DD, Ward EG, Kinsey AW, Simonavice E, Panton LB, Kim JS: The effects of six weeks of supplementation with multi-ingredient performance supplements and resistance training on anabolic hormones, body composition, strength, and power in resistance-trained men. J Int Soc Sports Nutr 2012, 9:49.

22. Willoughby DS, Stout JR, Wilborn CD: Effects of resistance training and protein plus amino acid supplementation on muscle anabolism, mass, and strength. Amino Acids 2007, 32:467-477.

23. Schmitz SM, Hofheins JE, Lemieux R: Nine weeks of supplementation with a multi-nutrient product augments gains in lean mass, strength, and muscular performance in resistance trained men. $J$ Int Soc Sports Nutr 2010, 7:40.

24. Sheikholeslami Vatani D, Ahmadi S, Ahmadi Dehrashid K, Gharibi F: Changes in cardiovascular risk factors and inflammatory markers of young, healthy, men after six weeks of moderate or high intensity resistance training. J Sports Med Phys Fitness 2011, 51:695-700.

25. Cornelissen VA, Fagard RH, Coeckelberghs E, Vanhees L: Impact of resistance training on blood pressure and other cardiovascular risk factors: a meta-analysis of randomized, controlled trials. Hypertension 2011, 58:950-958.

26. Bird SP, Tarpenning KM, Marino FE: Effects of liquid carbohydrate/essential amino acid ingestion on acute hormonal response during a single bout of resistance exercise in untrained men. Nutrition 2006, 22:367-375.

27. Sharp CP, Pearson DR: Amino acid supplements and recovery from high-intensity resistance training. J Strength Cond Res 2010, 24:1125-1130.

28. Gkaliagkousi E, Ferro A: Nitric oxide signalling in the regulation of cardiovascular and platelet function. Front Biosci 2011, 16:1873-1897.

29. Orea-Tejeda A, Orozco-Gutierrez JJ, Castillo-Martinez L, Keirns-Davies C, Montano-Hernandez P, Vazquez-Diaz O, Valdespino-Trejo A, Infante O, Martinez-Memije R: The effect of L-arginine and citrulline on endothelial function in patients in heart failure with preserved ejection fraction. Cardiol J 2010, 17:464-470.

30. Franks AM, Schmidt JM, McCain KR, Fraer M: Comparison of the effects of energy drink versus caffeine supplementation on indices of 24-hour ambulatory blood pressure. Ann Pharmacother 2012, 46:192-199.

31. Alvares TS, Conte CA, Paschoalin VM, Silva JT, Meirelles Cde M, Bhambhani YN, Gomes PS: Acute l-arginine supplementation increases muscle blood volume but not strength performance. Appl Physiol Nutr Metab 2012, 37:115-126.

32. Kadoglou NP, Fotiadis G, Athanasiadou Z, Vitta I, Lampropoulos S, Vrabas IS: The effects of resistance training on $A p o B / A p o A-I$ ratio, $L p(a)$ and inflammatory markers in patients with type 2 diabetes. Endocrine 2012, 42:561-569. 
33. McCormack JP, Allan GM: Measuring hsCRP-an important part of a comprehensive risk profile or a clinically redundant practice? PLoS Med 2010, 7:e1000196.

34. Freedman ND, Park Y, Abnet CC, Hollenbeck AR, Sinha R: Association of coffee drinking with total and cause-specific mortality. N Engl J Med 2012, 366:1891-1904.

35. Bresciani G, Cuevas MJ, Molinero O, Almar M, Suay F, Salvador A, de Paz JA, Marquez S, Gonzalez-Gallego J: Signs of overload after an intensified training. Int I Sports Med 2011, 32:338-343.

36. Baechle TR ER: Essentials of strength and conditioning. 3rd edition. Human Kinetics; 2008.

doi:10.1186/1743-7075-10-39

Cite this article as: Ormsbee et al:: The effects of pre- and post-exercise consumption of multi-ingredient performance supplements on cardiovascular health and body fat in trained men after six weeks of resistance training: a stratified, randomized, double-blind study. Nutrition \& Metabolism 2013 10:39.

\section{Submit your next manuscript to BioMed Central and take full advantage of:}

- Convenient online submission

- Thorough peer review

- No space constraints or color figure charges

- Immediate publication on acceptance

- Inclusion in PubMed, CAS, Scopus and Google Scholar

- Research which is freely available for redistribution 